\title{
Abelian Dirac-type monopoles and the dual Meissner effect in QCD
}

\author{
Atsuki Hiraguchi ${ }^{1,2, *}$, Katsuya Ishiguro $^{3, * *}$, and Tsuneo Suzuki ${ }^{4, * * *}$ \\ ${ }^{1}$ Institute of Physics, National Yang Ming Chiao Tung University, \\ Hsinchu 30010, Taiwan \\ ${ }^{2}$ Department of Mathematics and Physics, Kochi University, \\ Kochi 780-8520, Japan \\ ${ }^{3}$ Library and Information Technology, Kochi University, \\ Kochi 780-8520, Japan \\ ${ }^{4}$ RCNP, Osaka University, Ibaraki Osaka 567-0047, Japan
}

\begin{abstract}
We investigate the Abelian dual Meissner effect due to violation of the non-Abelian Bianchi identity in $S U(3)$ gauge thoery without gauge fixing. To decide the vacuum type, we evaluate the Ginzburg-Landau parameter from the spatial distribution of color electric fields and squared monopole density. Although the study is done only on $24(40)^{3} \times 4$ lattice at $\beta=5.6$, the $S U(3)$ vacuum is found to be of the type 1 near the border of type 1 and type 2 . We also confirm the dual Ampère's law directly.
\end{abstract}

\section{Introduction}

Quark confinement mechanism is one of the most important problem in particle physics [1]. A dual superconducting picture [2,3] as an idea of color confinement mechanism, has been investigated for many years. In this picture, quarks are confined by condensation of QCD monopoles. Although the idea of the dual superconducting picture is very interesting in understanding the mechanism of confinement, in constract to SUSY QCD [4] or GeorgiGlashow model [5, 6] with scalar fields, it is not straightforward to define monopoles in QCD. Intoduction of a partial gauge fixing [7] projecting QCD to its maximal Abelian torus group, is one interesting idea to define monopoles in QCD but there are infinte ways of such gauge fixings. Numerically the maximally Abelian gauge $[8,9]$, which is one of the Abelian projections, seems to support the conjecture [10,11].

Recently one of the authors (T.S.) found that the violation of the non-Abelian Bianchi identity(VNABI) is equal to Abelian-like monopoles in the continuum theory [12]. This idea is similar to the Dirac monopole [13] in U(1) quantum electrodynamics. It is confirmed that VNABI has the continuum limit in pure $S U(2)$ gauge theory $[14,15]$. Therefore showing the continuum limit of VNABI in $S U(3)$ gauge theory is very important. Recent studies about the monopole dominance in $S U(3)$ are presented in another report [16] in this conference. Here, we discuss the Abelian dual Meissner effect due to VNABI in $S U(3)$ gauge theory.

\footnotetext{
*e-mail: a.hiraguchi@nycu.edu.tw

**e-mail: ishiguro@kochi-u.ac.jp

***e-mail: tsuneo@rcnp.osaka-u.ac.jp
} 
Evaluating the spatial distribution of color electric fields and monopoles has been investigated in $S U(2)$ gauge theory. Especially, in Ref. [17], they investigated the dual Meissner effect in $S U(2)$ gauge theory without any gauge fixing. They confirmed the vacuum type is near the border between type 1 and type 2 and also the dual Ampère's law is satisfied. The Abelian monopole current squeezes the Abelian color electric field as a solenoidal current. These results agree with the dual superconducting picture. Our purpose of this work is to confirm the dual Meissner effect due to VNABI and to determine the vacuum type in $S U(3)$. In this report, first we explain the lattice definition of monopoles due to VNABI briefly and second show the results of the Abelian dual Meissner effect. Especially, we show the results of the Ginzburg-Landau parameter (GL) and the dual Ampère's law, although at one gauge coupling constant in the present stage.

\section{VNABI}

We explain the definiton of monopoles due to VNABI in this section briefly. Details of the derivation are shown in Ref.[16].

If gauge fields have a line singularity in the continuum QCD, the non-Abelian Bianchi identiry is violated. Then the violation (VNABI) corresponds to Abelian-like monopoles. In the case of $S U(3)$ gauge theory, VNABI is regarded as eight Abelian-like monopoles in the continuum QCD. The VNABI can be written as

$$
D_{\nu} G_{\mu \nu}^{*}=\partial_{\nu} f_{\mu \nu}^{*}
$$

where $D_{\mu}=\partial_{\mu}-i g A_{\mu}$ is a covariant derivative, $G_{\mu \nu}$ is a non-Abelian field strength and $f_{\mu \nu}$ is defined as $f_{\mu v}=\partial_{\mu} A_{v}-\partial_{v} A_{\mu}=\left(\partial_{\mu} A_{v}^{a}-\partial_{v} A_{\mu}^{a}\right) \lambda^{a} / 2$. In the case of $S U(3), \lambda^{a}$ are the Gell-Mann matrices.

In $S U(3)$ QCD on lattice, it is not straightforward to define the monopole. To extract Abelian link fields for all eight colors separately from non-Abelian gauge field matrix is not simple, since in $S U(3)$ the non-Abelian gauge field is not expanded by the Lie-Algebra elements in a simple way as in $S U(2)$. We use the following method to define the Abelian link field by maximizing the following overlap quantity by taking a functional derivative with respect to an Abelian link field $\theta_{\mu}^{a}(s)$ :

$$
R A=\sum_{s, \mu} \operatorname{Tr}\left(e^{i \theta_{\mu}^{a}(s) \lambda^{a}} U_{\mu}^{\dagger}(s)\right)
$$

This choice in $S U(2)$ leads us to the same Abelian link fields adopted in Ref. [17].

For example, we get from the maximization condition of (2) an Abelian link field $\theta_{1}(s, \mu)$ corresponding to $\sigma_{1}(S U(2))$ and $\lambda_{1}(S U(3))$ as

$$
\begin{aligned}
\theta_{1}(s, \mu) & =\tan ^{-1} \frac{U_{1}(s, \mu)}{U_{0}(s, \mu)}, \quad\left(\mathrm{SU} 2: \quad U(s, \mu)=U_{0}(s, \mu)+i \vec{\sigma} \cdot \vec{U}(s, \mu)\right) \\
& =\tan ^{-1} \frac{\operatorname{Im}\left(U_{12}(s, \mu)+U_{21}(s, \mu)\right)}{\operatorname{Re}\left(U_{11}(s, \mu)+U_{22}(s, \mu)\right)}, \quad(\mathrm{SU} 3)
\end{aligned}
$$

Once Abelian link variables are fixed, we can extract Abelian, monopole and photon parts from the Abelian plaquette variable as follows:

$$
\theta_{\mu \nu}^{a}(s)=\bar{\theta}_{\mu \nu}^{a}(s)+2 \pi n_{\mu \nu}^{a}(s)\left(\left|\bar{\theta}_{\mu \nu}^{a}\right|<\pi\right),
$$

where $n_{\mu \nu}^{a}(s)$ is an integer corresponding to the number of the Dirac string. Then an Abelian monopole current is defined by

$$
k_{\mu}^{a}(s)=-(1 / 2) \epsilon_{\mu \alpha \beta \gamma} \partial_{\alpha} \bar{\theta}_{\beta \gamma}^{a}(s+\hat{\mu})=(1 / 2) \epsilon_{\mu \alpha \beta \gamma} \partial_{\alpha} n_{\beta \gamma}^{a}(s+\hat{\mu})
$$

This definition (4) satisfies the Abelian conservation condition and takes an integer value which corresponds to the magnetic charge obeying the Dirac quantization condition [18]. 


\section{The Abelian dual Meissner effect in $S U(3)$}

\subsection{Simulation details of the flux-tube profile}

In this section, we show the results with respect to the Abelian dual Meissner effect. In the previous work [17] studying the spatial distribution of color electric fields and monopole currents, they used the connected correlations between a non-Abelian Wilson loop and Abelian operators in $S U(2)$ gauge theory without gauge fixing. We apply the same method to $S U(3)$ gauge theory without gauge fixing. Here we employ the standard Wilson action on the $24^{3}\left(40^{3}\right) \times 4$ lattice with the coupling constant $\beta=5.6$. We consider a finite temperature system at $T=0.8 T_{c}$. To improve the signal-to-noise ratio, the APE smearing is applied to the spatial links and the hypercubic blocking is applied to the temporal links. We introduce random gauge transformations to improve the signal to noise ratios of the data concerning the Abelian operators.

To measure the flux-tube profiles, we consider a connected correlation functions as done in $[19,20]$ :

$$
\rho_{\text {conn }}(O(r))=\frac{\left\langle\operatorname{Tr}\left(P(0) L O(r) L^{\dagger}\right) \operatorname{Tr} P^{\dagger}(d)\right\rangle}{\left\langle\operatorname{Tr} P(0) \operatorname{Tr} P^{\dagger}(d)\right\rangle}-\frac{1}{3} \frac{\left\langle\operatorname{Tr} P(0) \operatorname{Tr} P^{\dagger}(d) \operatorname{Tr} O(r)\right\rangle}{\left\langle\operatorname{Tr} P(0) \operatorname{Tr} P^{\dagger}(d)\right\rangle},
$$

where $P$ denotes a non-Abelian Polyakov loop, $L$ indicates a Schwinger line, $r$ is a distance from a flux-tube and $d$ is a distance between Polyakov loops. We use the cylindrical coordinate $(r, \phi, z)$ to parametrize the $q-\bar{q}$ system as shown in Fig 1 .

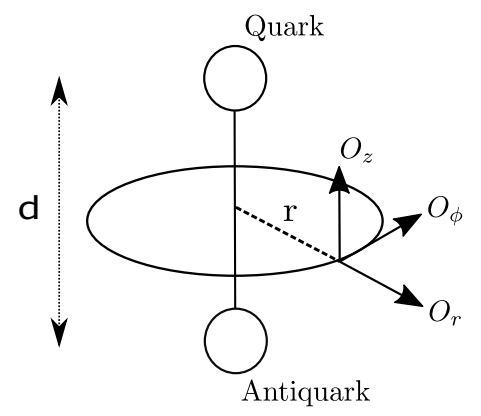

Figure 1. The definition of the cylindrical coordinate $(r, \phi, z)$ along the $q-\bar{q}$ axis. The $d$ corresponds to the distance between Polyakov loops.

\subsection{The spatial distribution of color electric fields}

First of all, we show the results of Abelian color electric fields using an Abelian gauge field $\theta_{1}(s, \mu)$. To evaluate the Abelian color electric field, we adopt the Abelian plaquette as an operator $O(r)$. We calculate a penetration length $\lambda$ from the Abelian color electric fields for $d=3,4,5,6$ at $\beta=5.6$ and check the $d$ dependence of $\lambda$. To improve the accuracy of the fitting, we evaluate $O(r)$ at both on-axis and off-axis distances. As a result, we find the Abelian color electric fields $E_{z}^{A}$ alone are squeezed as in Fig 2. We fit these results to a fitting function,

$$
f(r)=c_{1} \exp (-r / \lambda)+c_{0}
$$

The parameter $\lambda$ corresponds to the penetration length. We summarize the values of parameters in Table 1. We find the values of the penetration length are almost the same. 


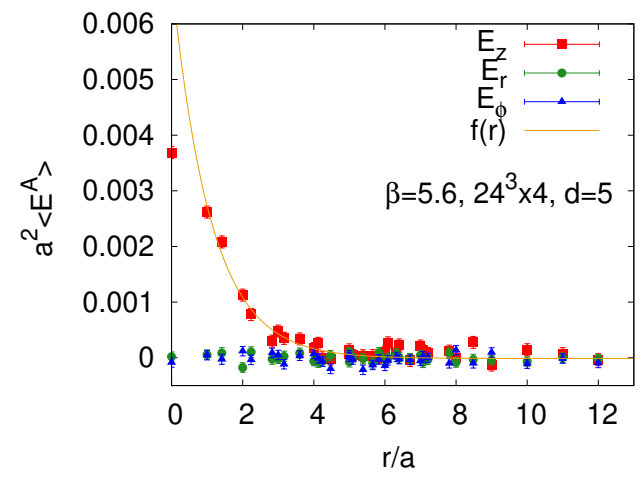

Figure 2. The Abelian color electric field with $d=5$ at $\beta=5.6$ on $24^{3} \times 4$ lattices.

Table 1. The penetration length $\lambda$ at $\beta=5.6$ on $24^{3} \times 4$ lattices.

\begin{tabular}{|c|c|c|c|c|}
\hline$d$ & $\lambda / a$ & $c_{1}$ & $c_{0}$ & $\chi^{2} / N_{d f}$ \\
\hline 3 & $0.91(1)$ & $0.0100(2)$ & $-0.000002(8)$ & 1.31628 \\
\hline 4 & $1.10(6)$ & $0.0077(4)$ & $-0.00005(4)$ & 0.972703 \\
\hline 5 & $1.09(8)$ & $0.0068(6)$ & $-0.00001(4)$ & 0.995759 \\
\hline 6 & $1.1(1)$ & $0.0055(8)$ & $-0.00008(7)$ & 0.869692 \\
\hline
\end{tabular}

\subsection{The spatial distribution of monopole currents}

Next we show the result of the spatial distribution of Abelian-like monopole currents. We define the Abelian-like monopole currents on the lattice as in Eq. (4). In this study we evaluate the connected correlation (5) between $k^{1}$ and the two non-Abelian Polayakov loops. We use random gauge transformations to evaluate this correlation. As a result, we find the spatial distribution of monopole currents around the flux-tube at $\beta=5$.6. Only the monopole current in the azimuthal direction, $k_{\phi}^{1}$, shows the correlation with the two non-Abelian Polyakov loops.

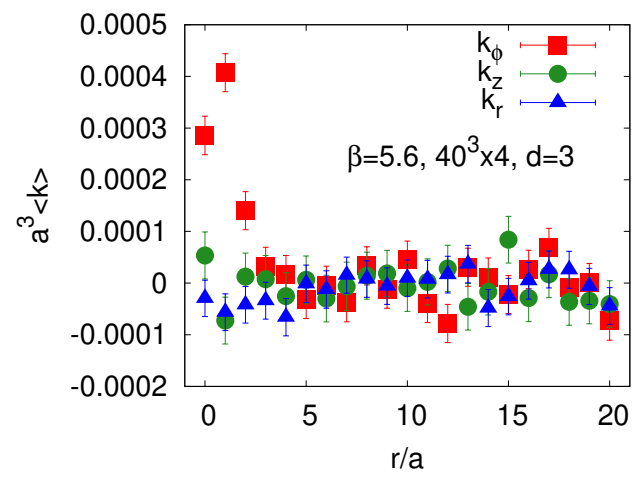

Figure 3. The monopole current at $\beta=5.6$ on $40^{3} \times 4$ lattices. 


\subsection{The dual Ampère's law}

In previous $S U(2)$ researches [17], they investigated the dual Ampère's law to see what squeezes the color-electric field. In the case of $S U(2)$ gauge theory without gauge fixings, they confirmed the dual Ampère's law and the monopole currents squeeze the color-electric fields. In this section we show the results of the dual Ampère's law in the case of $S U(3)$ gauge theory. The definition of monopole currents gives us the following relation,

$$
\left(\operatorname{rot} E^{a}\right)_{\phi}=\partial_{t} B_{\phi}^{a}+2 \pi k_{\phi}^{a},
$$

where index $a$ is a color index.

As a results, we confirm that there is no signal of the magnetic displacement current $\partial_{t} B_{\phi}^{a}$ around the flux-tube for $d=3$ at $\beta=5.6$ as shown in Fig. 4. It suggests that the Abelian-like monopole current squeezes the Abelian color electric field as a solenoidal current in $S U(3)$ gauge theory without gauge fixing, although more data for larger $d$ are necessary.

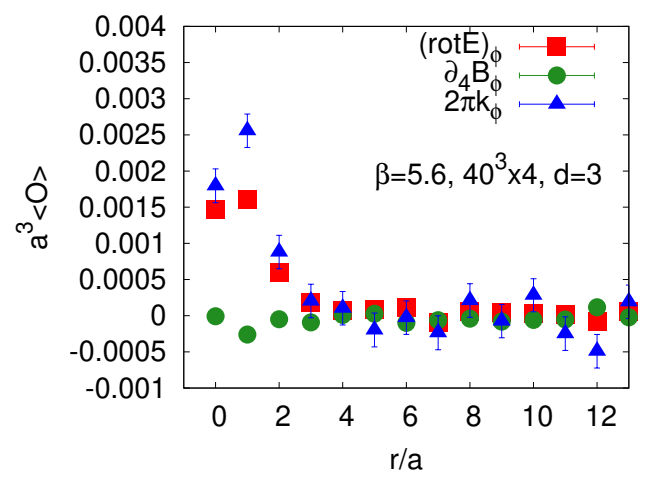

Figure 4. The dual Ampère's law at $\beta=5.6$ on $40^{3} \times 4$ lattices.

\subsection{The vacuum type in $S U(3)$ gauge theory without gauge fixing}

Finally, we evaluate the Ginzburg-Landau parameter, which characterizes the type of the (dual) superconducting vacuum. In the previous result [17], they found that the vacuum type is near the border between type 1 and type 2 dual superconductors by using the $S U(2)$ gauge theory without gauge fixing. We apply the same method to $S U(3)$ gauge theory.

To evaluate the coherence length, we measure the correlation between the squared monopole density and two non-Abelian Polyakov loops by using the disconnected correlation function $[17,21]$,

$$
\left\langle k^{2}(r)\right\rangle_{q \bar{q}}=\frac{\left\langle\operatorname{Tr} P(0) \operatorname{Tr} P^{\dagger}(d) \sum_{\mu, a} k_{\mu}^{a}(r) k_{\mu}^{a}(r)\right\rangle}{\left\langle\operatorname{Tr} P(0) \operatorname{Tr} P^{\dagger}(d)\right\rangle}-\left\langle\sum_{\mu, a} k_{\mu}^{a}(r) k_{\mu}^{a}(r)\right\rangle .
$$

We fit the profiles to the function,

$$
g(r)=c_{1}^{\prime} \exp \left(-\frac{\sqrt{2} r}{\xi}\right)+c_{0}^{\prime}
$$


where the parameter $\xi$ corresponds to the coherence length. We plot the profiles of $\left\langle k^{2}(r)\right\rangle_{q \bar{q}}$ in Fig 5. As a result, we could evaluate the coherence length $\xi$ for $d=3,4,5,6$ at $\beta=5.6$ and find almost the same values of $\xi / \sqrt{2}$ for each $d$ as shown in Table 2. Using these parameters $\lambda$ and $\xi$, we could evaluate the Ginzburg-Landau parameter. The GL parameter $\kappa=\lambda / \xi$ can be defined as the ratio of the penetration length and the coherence length. If $\sqrt{2} \kappa<1$, the vacuum type is of the type 1 and if $\sqrt{2} \kappa>1$, the vacuum is of the type 2 . We show the GL parameters in $S U(3)$ gauge theory in Table 3 . We find that the vacuum is of the type 1 near the border between type 1 and type 2, although the study is done at one gauge coupling constant $\beta=5.6$. This is the first result of the vacuum type in pure $S U(3)$ gauge theory without gauge fixing, although different $\beta$ data are necessary to show the continuum limit.

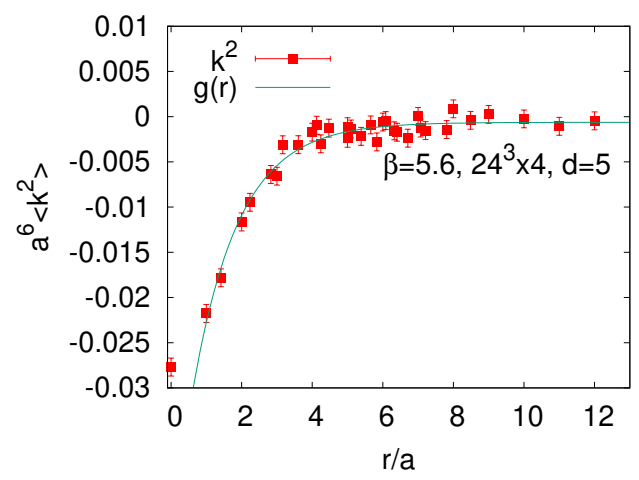

Figure 5. The squared monopole density with $d=5$ at $\beta=5.6$ on $24^{3} \times 4$ lattices.

Table 2. The coherence length $\xi / \sqrt{2}$ at $\beta=5.6$ on $24^{3} \times 4$ lattices.

\begin{tabular}{|c|c|c|c|c|}
\hline$d$ & $\xi / \sqrt{2} a$ & $c_{1}^{\prime}$ & $c_{0}^{\prime}$ & $\chi^{2} / N_{d f}$ \\
\hline 3 & $1.04(6)$ & $-0.050(3)$ & $0.0001(2)$ & 0.997362 \\
\hline 4 & $1.17(7)$ & $-0.052(3)$ & $-0.0003(2)$ & 1.01499 \\
\hline 5 & $1.3(1)$ & $-0.047(3)$ & $-0.0006(3)$ & 0.99758 \\
\hline 6 & $1.1(1)$ & $-0.052(8)$ & $-0.0013(5)$ & 1.12869 \\
\hline
\end{tabular}

Table 3. The Ginzburg-Landau parameters at $\beta=5.6$ on $24^{3} \times 4$ lattice.

\begin{tabular}{|c|c|}
\hline $\mathrm{d}$ & $\sqrt{2} \kappa$ \\
\hline 3 & $0.87(5)$ \\
\hline 4 & $0.93(7)$ \\
\hline 5 & $0.83(9)$ \\
\hline 6 & $0.9(2)$ \\
\hline
\end{tabular}




\section{ACKNOWLEDGMENTS}

The numerical simulations of this work were done using High Performance Computing resources at Cybermedia Center and Research Center for Nuclear Physics of Osaka University, at Cyberscience Center of Tohoku University and at KEK. The authors would like to thank these centers for their support of computer facilities. T.S was finacially supported by JSPS KAKENHI Grant Number JP19K03848.

\section{References}

[1] K. Devlin, The millennium problems : the seven greatest unsolved mathematical puzzles of our time,Basic Books, New York (2002).

[2] G. 't Hooft, in Proceedings of the EPS International, edited by A. Zichichi, p. 1225, 1976.

[3] S. Mandelstam, Phys. Rept. 23, 245 (1976).

[4] N. Seiberg and E. Witten, Nucl. Phys. B426, 19 (1994).

[5] G. 't Hooft, Nucl. Phys. B79, 276 (1974).

[6] A. M. Polyakov, Nucl. Phys. B120, 429 (1977).

[7] G. 't Hooft, Nucl. Phys. B190, 455 (1981).

[8] A. S. Kronfeld, M. L. Laursen, G. Schierholz, and U. J. Wiese, Phys. Lett. B198, 516 (1987).

[9] A. S. Kronfeld, G. Schierholz, and U. J. Wiese, Nucl. Phys. B293, 461 (1987).

[10] T. Suzuki, Nucl. Phys. Proc. Suppl. 30, 176 (1993).

[11] M. N. Chernodub and M. I. Polikarpov, in "Confinement, Duality and Nonperturbative Aspects of QCD", edited by P. van Baal, p. 387, Cambridge, 1997, Plenum Press.

[12] Tsuneo Suzuki, A new scheme for color confinement due to violation of the non-Abelian Bianchi identities, arXiv:1402.1294

[13] P. Dirac, Proc. Roy. Soc. (London) A 133, 60 (1931).

[14] Tsuneo Suzuki, Katsuya Ishiguro and Vitaly Bornyakov, Phys. Rev. D97, 034501 (2018). Erratum: Phys. Rev. D97, 099905 (2018).

[15] Tsuneo Suzuki, Phys. Rev. D 97034509 (2018).

[16] Tsuneo Suzuki, Atsuki Hiraguchi and Katsuya Ishiguro, "Monopoles of the Dirac type and color confinement in QCD" in Proceeding of vConf 21.

[17] T. Suzuki, M. Hasegawa, K. Ishiguro, Y. Koma and T. Sekido, Phys. Rev. D80, 054504 (2009).

[18] T. A. DeGrand and D. Toussaint, Phys. Rev. D22, 2478 (1980).

[19] Paolo Cea, Leonardo Cosmai, Francesca Cuteri, and Alessandro Papa, Journal of High Energy Physics, 2016, 6 (2016).

[20] Paolo Cea, Leonardo Cosmai, Francesca Cuteri, and Alessandro Papa, EPJ Web Conf., 175, 12006 (2018).

[21] M. N. Chernodub, Katsuya Ishiguro, Yoshihiro Mori, Yoshifumi Nakamura, M. I. Polikarpov, Toru Sekido, Tsuneo Suzuki, and V. I. Zakharov, Phys. Rev. D72, 074505 (2005). 